\title{
Caracterización radar de la supercélula tornádica de larga duración del 2 de octubre de 2013 en Castilla y León
}

\author{
José Antonio Quirantes Calvo ${ }^{1}$ (jquirantesc@aemet.es) \\ Alicia López Rejas' (alopezr@aemet.es) \\ José Miguel Gutiérrez Núñez ${ }^{1}$ (jgutierrezn@aemet.es) \\ Álvaro Subías Díaz-Blanco ${ }^{1}$ (asubiasd@aemet.es) \\ Cecilia Marcos Martín11 (cmarcosm@aemet.es) \\ Juan Pablo Álvarez Alonso² (jalvareza@aemet.es) \\ José Antonio Gallego Poveda ${ }^{3}$ (josesaja@yahoo.es)
}

\author{
${ }^{1}$ AEMET / Dirección de Producción e Infraestructuras \\ ${ }^{2}$ AEMET / Delegación Territorial en Castilla y León \\ ${ }^{3}$ Fotógrafo, cazatormentas y socio de la AME
}

\begin{abstract}
RESUMEN
El objeto del trabajo es la caracterización radar de una célula convectiva, se concluirá que se trató de una supercélula tornádica de larga duración.
\end{abstract}

PALABRAS CLAVE: supercélula; radar; mesociclón; granizo; tornado; viento Doppler.

\section{INTRODUCCIÓN}

El 2 de octubre de 2013 a las 14:30 Z se formó una estructura convectiva al SW de Zamora que se desplazó hacia el ENE, cruzando las provincias de Zamora, Valladolid, Palencia y Burgos, disipándose en el País Vasco sobre las 21-22 Z, recorriendo en total unos $300 \mathrm{~km}$ en unas 7 horas. A su paso, se registraron efectos adversos de gran severidad, tales como dos tornados (uno de ellos posiblemente F2 al norte de Valladolid), granizo del tamaño de pelotas de tenis (puntos de Palencia y Burgos), e inundaciones rápidas. El objetivo principal de este trabajo es realizar la caracterización radar de la tormenta. Se comprobará que esta se originó a partir de un storm-splitting del que solo progresó el miembro ciclónico cuya evolución dio lugar a una supercélula tornádica de duración excepcional. Aunque las supercélulas no son raras en la península ibérica, sí lo son aquellas con duraciones de más de 4-5 horas, estando la media en torno a las 1-2 horas. Se incluye un resumen de los sondeos y del entorno preconvectivo, imágenes de satélite, descargas eléctricas y una colección de fotografías (figura 1), tanto de los daños en superficie como de la propia tormenta (José A. Gallego Poveda), en las que se pueden identificar algunas características visuales típicas de las supercélulas.

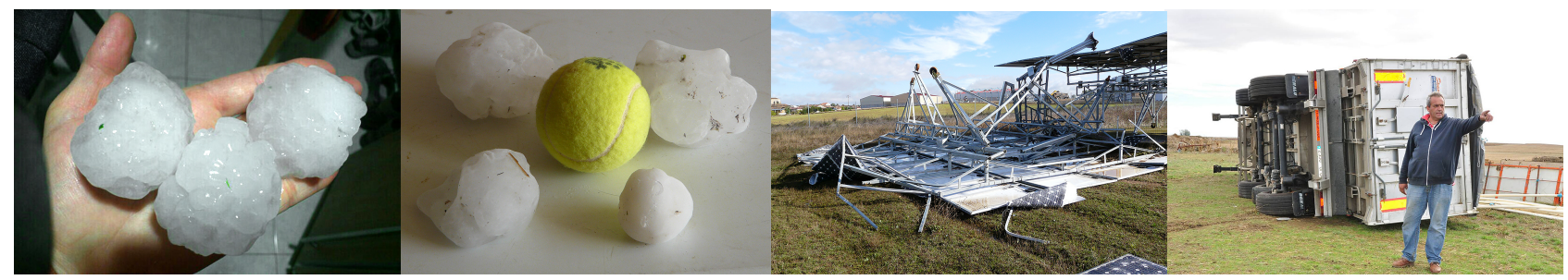

Figura 1 


\section{RESUMEN ENTORNO PRECONVECTIVO SINÓPTICO/MESOESCALAR}

La célula del estudio se desarrolló en un ambiente que reunía los ingredientes básicos para la formación de este tipo de estructuras. Por un lado, inestabilidad del perfil térmico con CAPE moderado, de alrededor de $500 \mathrm{~J} \mathrm{~kg}^{-1}$. Además, una vaguada acercándose hacia el oeste de la península ibérica aportando forzamiento dinámico que actuaría, junto con las convergencias en superficie, como mecanismo de disparo. Así mismo, con el acercamiento del chorro del SW, se obtuvo la cizalladura necesaria para la organización del sistema. También se observó en las estaciones de superficie de la zona, alta humedad en niveles bajos, último ingrediente necesario.

Se muestran a continuación algunos campos de los principales ingredientes que participaron en la formación de la supercélula:

- Forzamiento dinámico (figura 2a).

Amplia vaguada sobre el Atlántico al oeste de la península ibérica avanzando hacia el este.

$\mathrm{T}_{500 \mathrm{hPa}}=-13^{\circ} \mathrm{C}, \mathrm{V}_{300 \mathrm{hPa}}=65 \mathrm{kt}$ del SW (figura $2 \mathrm{~b}$ ).

- Inestabilidad convectiva moderada.

CAPE entre 500 y $1000 \mathrm{~J} \mathrm{~kg}^{-1}$ en toda la trayectoria de la supercélula (figura 2d).

- Perfil vertical del viento favorable a organizar supercélulas long-life.

CIZ08: $24 \mathrm{~m} \mathrm{~s}^{-1}$ y SHR3: $169 \mathrm{~m}^{2} \mathrm{~s}^{-2}$ (véanse sondeos).

- Niveles bajos: convergencia del viento en SFC y alto contenido en humedad.

Se observaron $\mathrm{Td}$ alrededor de $18^{\circ} \mathrm{C}$ y $\mathrm{HR}$ alrededor del $90 \%$ (figura $2 \mathrm{c}$ ).

a)

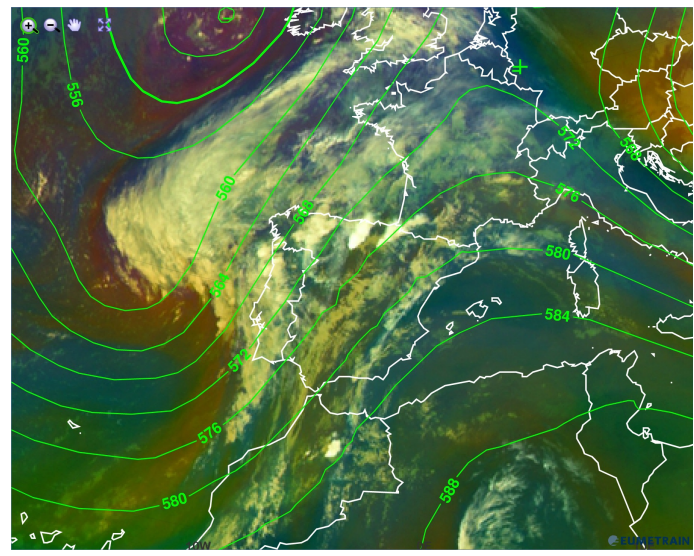

c)

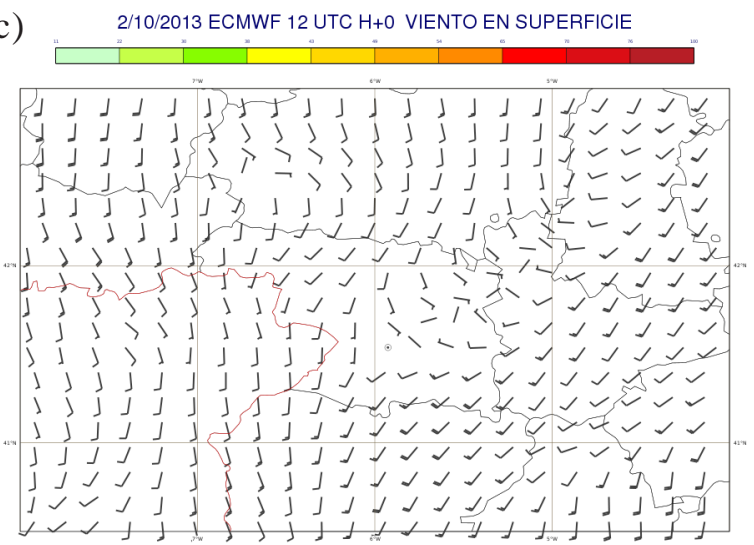

b) 2/10/2013 ECMWF 12 UTC H+3 ISOTACAS(m/s)+GEOPOTENCIAL 300

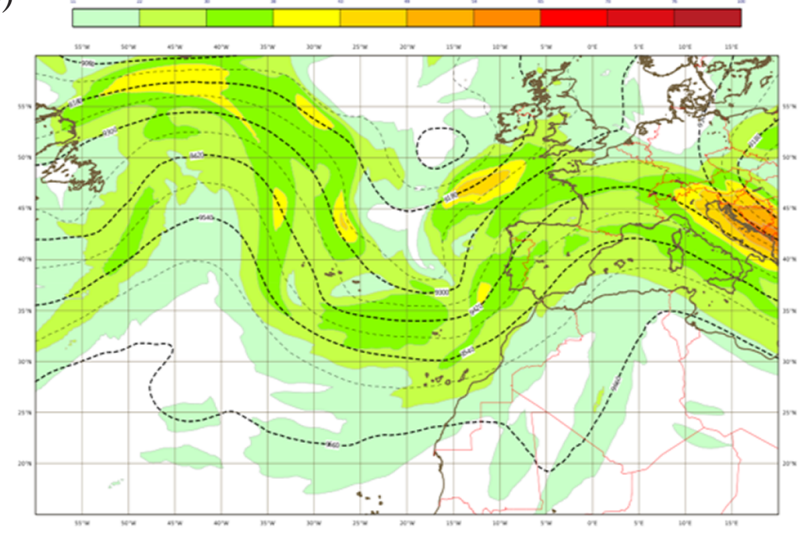

d)

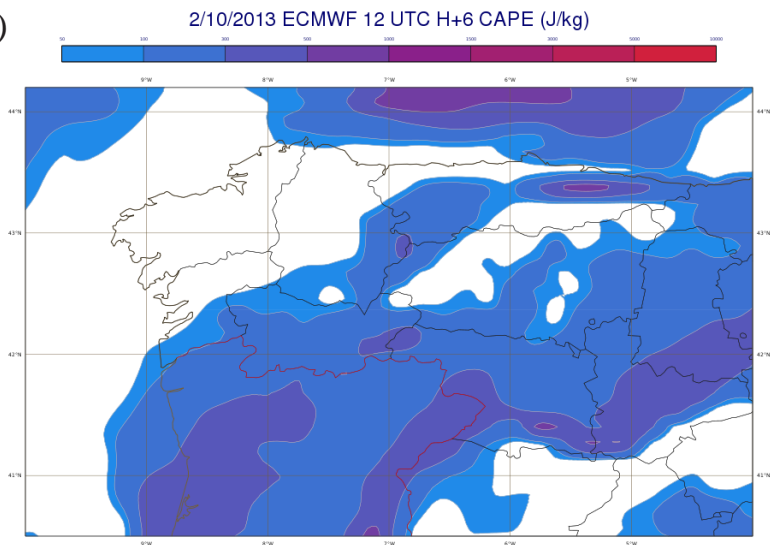

Figura 2 


\section{SONDEOS}

El análisis de los sondeos y de las hodógrafas del ambiente preconvectivo es una herramienta fundamental para determinar el tipo de sistema que podemos esperar en caso de que la convección se dispare. Lo ideal para analizar nuestro caso hubiese sido disponer de los sondeos observados en el punto en el cual se disparó nuestra célula y en el recorrido. Como no disponemos de ellos, hemos analizado someramente los sondeos previstos por el modelo HRES del ECMWF de la pasada más cercana a las horas de ocurrencia del fenómeno (véase la figura 3 ).

Zamora 14 Z $\left(\mathbf{4 1}^{\circ} \mathbf{2 4}^{\prime}\right.$ N, $5^{\circ}$ 42' W) - Formación: ligero giro horario de la hodógrafa en niveles bajos favorable al desarrollo del miembro «a derechas» (miembro ciclónico).

BURBUJA $100 \mathrm{hPa}$ : CAPEv 533,4 $\mathrm{J} \mathrm{kg}^{-1}$; NCAv 840,2 hPa.

CIZALLADURAS: CIZ08 47,6 kt; CIZ06 30,9 kt; CIZ01 13,4 kt.

HELICIDADES: SRH3 169,2 $\mathrm{m}^{2} \mathrm{~s}^{-2}$; ESRH 145,8 $\mathrm{m}^{2} \mathrm{~s}^{-2}$.

Palencia 17 Z $\left(\mathbf{4 1}^{\circ} 48^{\prime} \mathbf{N}, 5^{\circ}\right.$ 06’ W) - Tornado F2: giro horario de la hodógrafa en niveles bajos favorable al mantenimiento de la supercélula ciclónica.

BURBUJA $100 \mathrm{hPa}$ : CAPEv 714,4 J kg-1; NCAv 828,3 hPa.

CIZALLADURAS: CIZ08 58,1 kt; CIZ06 44,3 kt; CIZ01 16,4 kt.

HELICIDADES: SRH3 275,3 $\mathrm{m}^{2} \mathrm{~s}^{-2}$; ESRH $206,1 \mathrm{~m}^{2} \mathrm{~s}^{-2}$.

Burgos 18 Z (42 ${ }^{\circ} 18^{\prime} N, 3^{\circ} 48^{\prime}$ W) - Evolución 4 h: giro horario de la hodógrafa en niveles bajos favorable al mantenimiento de la supercélula ciclónica.

BURBUJA $100 \mathrm{hPa}$ : CAPEv 794,9 $\mathrm{J} \mathrm{kg}^{-1}$; NCAv 808,6 hPa.

CIZALLADURAS: CIZ08 52,3 kt; CIZ06 40,2 kt; CIZ01 18,6 kt.

HELICIDADES: SRH3 231,3 $\mathrm{m}^{2} \mathrm{~s}^{-2}$; ESRH $185,5 \mathrm{~m}^{2} \mathrm{~s}^{-2}$.
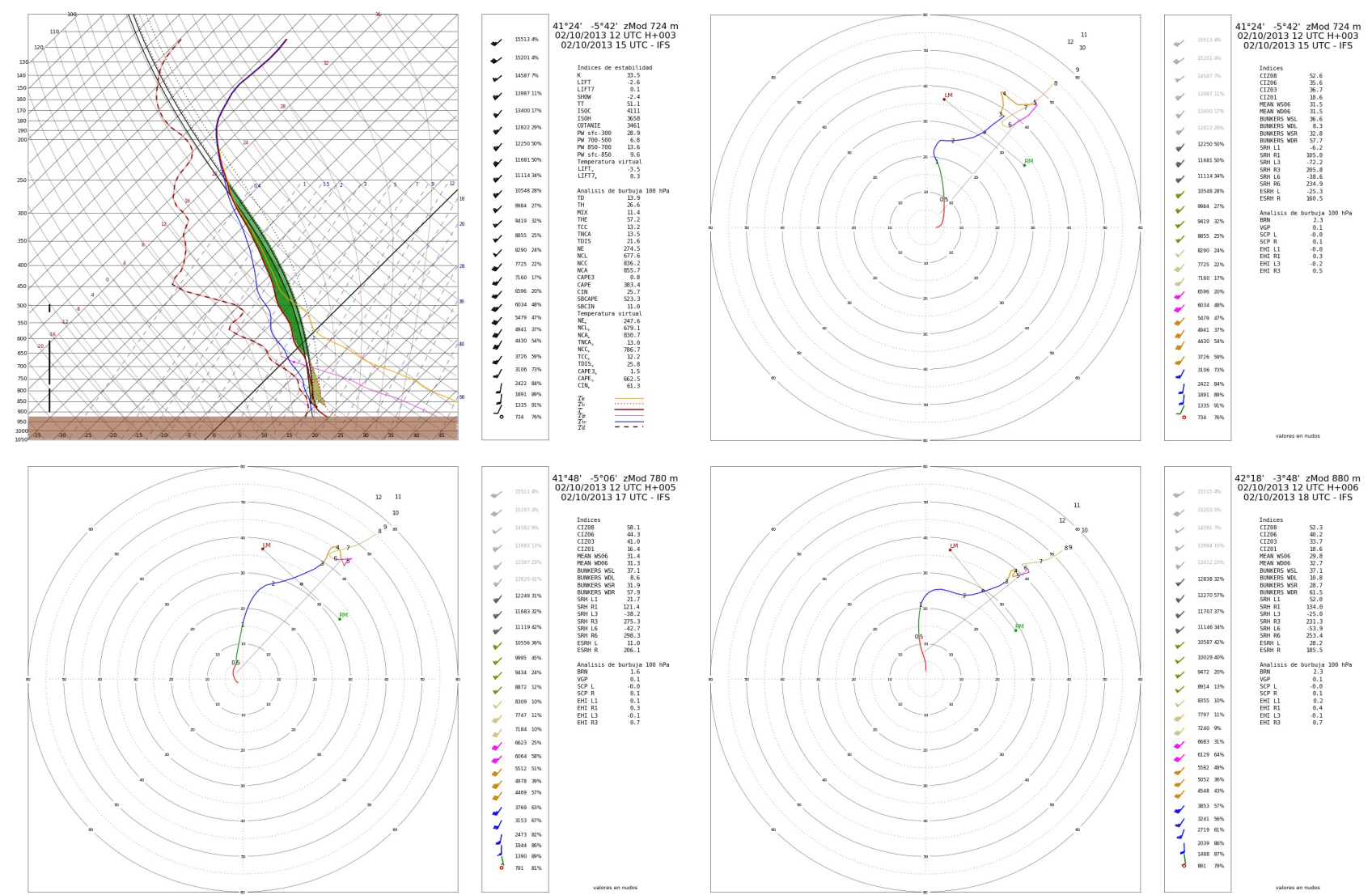

Figura 3 


\section{FOTOGRAFÍAS}

Las siguientes fotografías fueron realizadas por el fotógrafo y cazatormentas José Antonio Gallego Poveda desde varias localizaciones de la provincia de Palencia. En la imagen 4a se observa una vista general de la supercélula tomada al este de su posición. En ella, coincidiendo con el final de la carretera, se observa la base del mesociclón con una pequeña tail-cloud que apunta hacia la estela de precipitación de la FFD, situada a su derecha. La foto $4 \mathrm{~b}$ muestra a la derecha esa misma base del mesociclón, la tail-cloud y la FFD junto al margen derecho de la foto, mientras que a la izquierda del mesociclón aparece un gran claro asociado a la RFD. En la 4c se observan nubes bajas asociadas al frente de racha de la RFD. La 4d muestra el paso de ese frente de racha-RFD sobre la vertical del observador y por último la fotografía $4 \mathrm{e}$ es una toma nocturna iluminada por continuos rayos hacia el lado oeste de la supercélula, una vez se había adentrado en la provincia de Burgos, en ella se aprecia claramente el flanking-line.
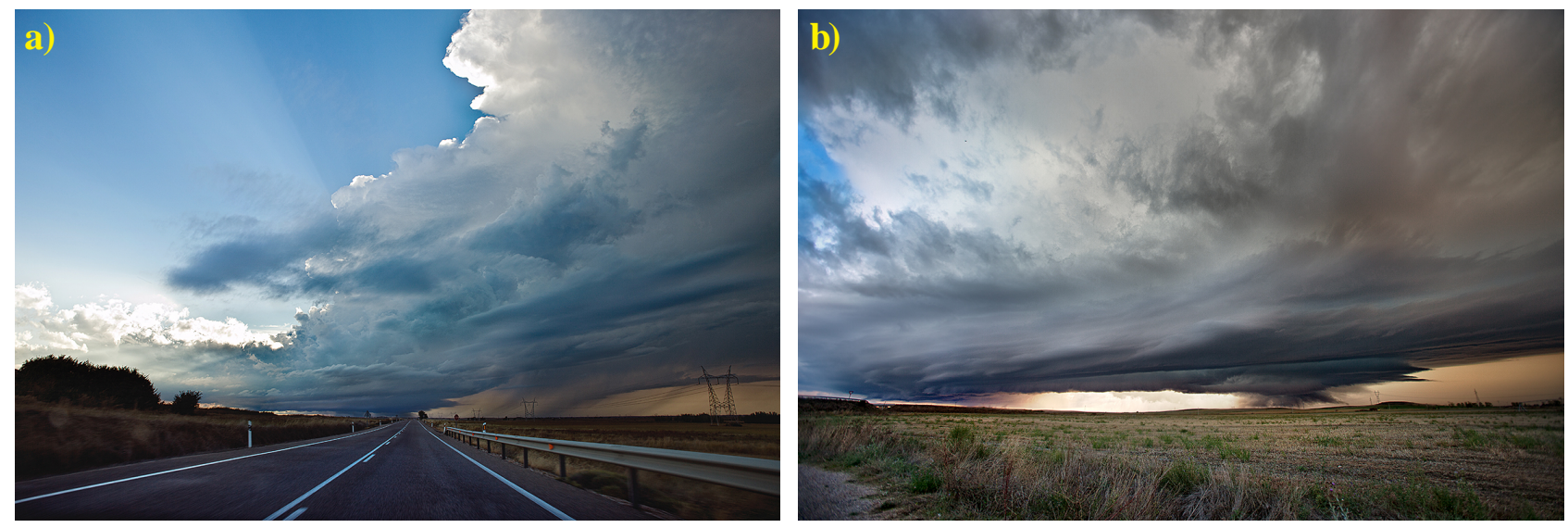

c)
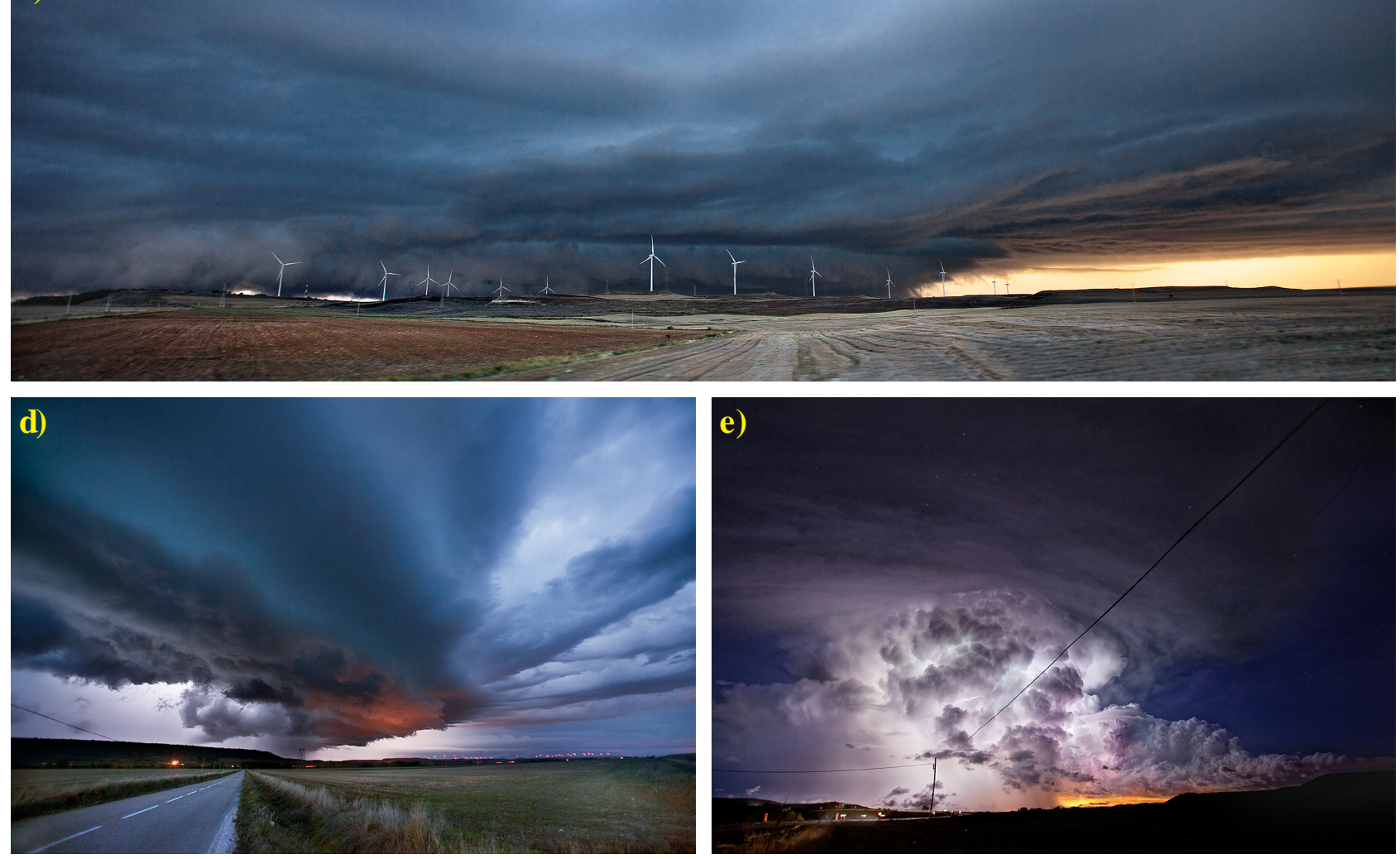

Figura 4 


\section{CARACTERIZACIÓN RADAR DE LA SUPERCÉLULA}

\subsection{Nota previa importante}

El radar de Palencia estuvo descalibrado durante la tormenta, provocando una subestimación en los valores de reflectividad. La empresa de mantenimiento revisó la cadena de recepción del radar tras este episodio encontrando que un cable defectuoso provocaba pérdidas de unos 7 a $8 \mathrm{dBz}$ en la señal, siendo sustituido. Se ha elegido una escala diferente de la operativa de AEMET para tener más variedad de colores y compensar algo este problema.

\subsection{Trayectoria}

Esta composición virtual muestra la evolución de la supercélula entre las 14:30Z y las 21:00 Z (véase la figura 5), extrayendo de cada ciclo radar solamente la franja de terreno donde se encontraba la tormenta. El objetivo es poder visualizar en un solo gráfico la evolución de la estructura a lo largo de todo su ciclo de vida. Se han elegido los CAPPI más representativos en cada caso. La célula convectiva inicial se forma al sur de otra tormenta previa no supercelular. El storm-splitting es visible hasta las 16:20Z. A partir de ahí, el miembro anticiclónico desaparece y queda solo la supercélula ciclónica. Se puede apreciar cómo el desvío hacia el este es más pronunciado a la hora en que se produjeron los tornados (uno entre 16:40Z y 17:00 Z, y otro entre 17:10Zy 17:40Z).

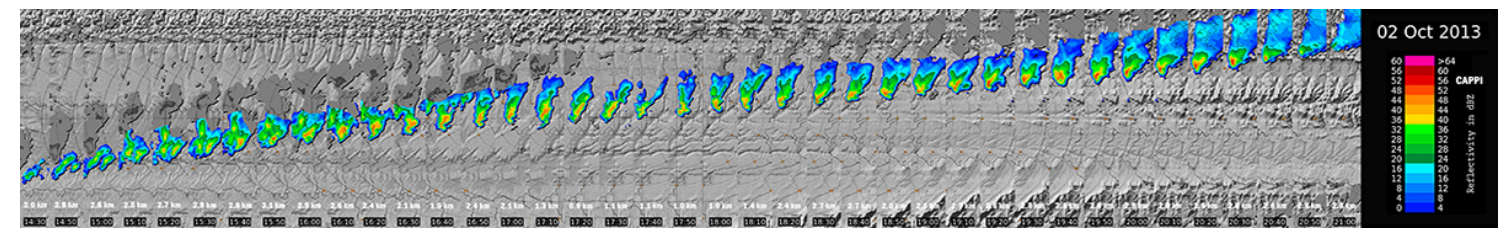

Figura 5

\subsection{Hook-Echo, WER, FFD y RFD}

La estructura en gancho es propia de los CAPPI bajos de las supercélulas y está originada por el giro del mesociclón al interactuar con el núcleo de precipitación (formado por la corriente descendente delantera o FFD y por la corriente descendente trasera o RFD), que se «enrollan» alrededor del mismo. En nuestro caso, al ser una supercélula ciclónica que se mueve hacia el ENE, la FFD queda posicionada en el arco situado al NE-NW-SW del mesociclón, y la RFD quedaría situada al SW-S-SE del mismo, es decir, el núcleo de precipitación es desplazado hacia sus flancos oeste (FFD) y sur (RFD) en forma de «seis», siendo la RFD la responsable de este último tramo. Al hueco del gancho se le llama WER (región de eco débil). En las figuras 6a, 6b y 6c se observa un «gancho» muy definido que coincide con el momento de la formación de uno de los dos tornados, que afectó entre

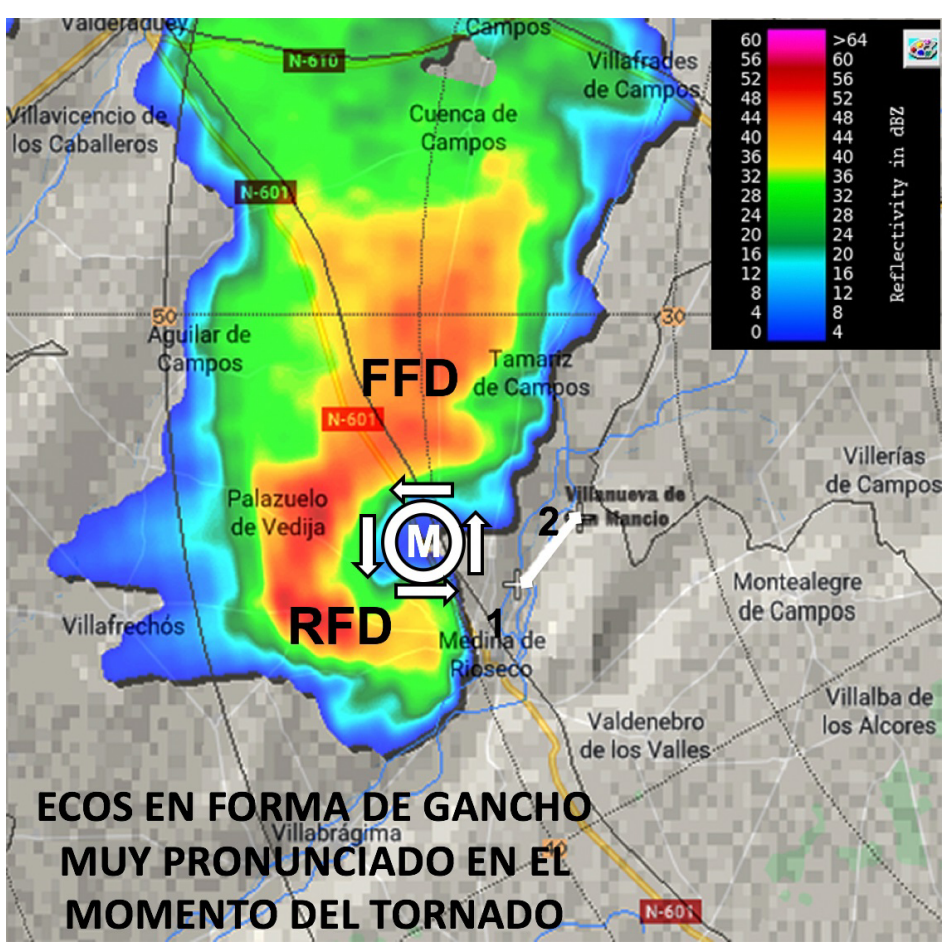

Figura 6.a 


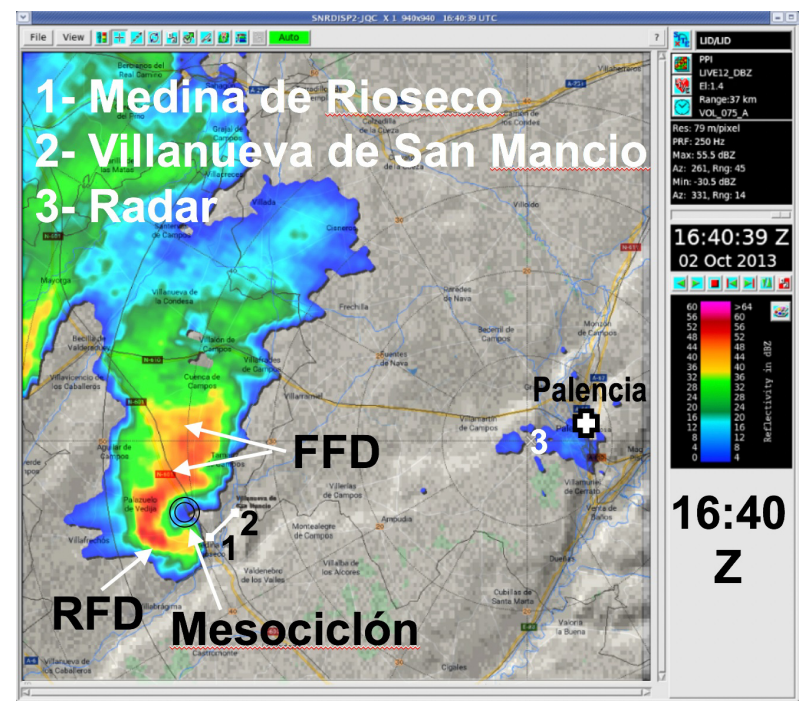

Figura 6b

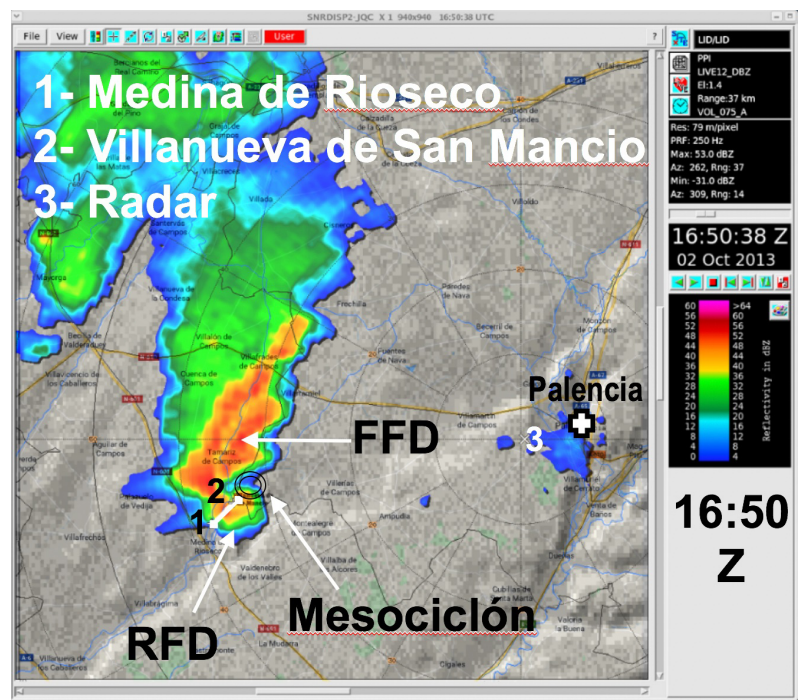

Figura 6c

16:40 Z y 17:00 Z a una línea de terreno de unos $4 \mathrm{~km}$ de longitud, orientada de $\mathrm{SW}$ a NE, entre las poblaciones de Medina de Rioseco (1) y Villanueva de San Mancio (2). La figura 6a es una ampliación de la figura 6b. [FFD = Forward Flank Downdraft, RFD = Rear Flank Downdraft, WER= Weak Echo Region $].$

\subsection{BWER-Vertical}

Es un mínimo local de reflectividad (Z) que se extiende desde niveles bajos a niveles medios y que está rodeado por Z más altas, excepto por debajo. Este «agujero» se produce porque la intensa corriente ascendente en rotación transporta muy eficientemente la precipitación hasta alturas muy grandes (12-15km), región donde es desplazada por el intenso flujo en niveles altos, quedando desacoplada en la vertical de las capas bajas y formando debajo de ella una cavidad sin ecos radar. Los dos cortes verticales son de las 16:40Z, momento del tornado: uno es perpendicular al movimiento de la tormenta (NW-SE) y otro paralelo (SW-NE). En ambos se aprecia un mínimo de $12 \mathrm{dBZ}$ a unos 4-5 km de altura situado debajo del máximo, 54-56 dBZ, a unos $8 \mathrm{~km}$ de altura (figuras 7a y 7b). [BWER = Bounded Weak Echo Region].

a)

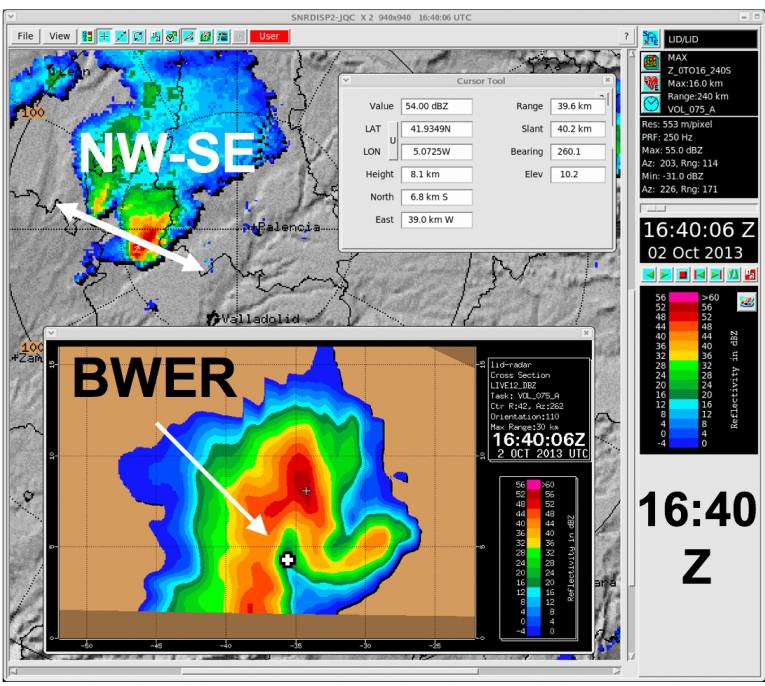

b)

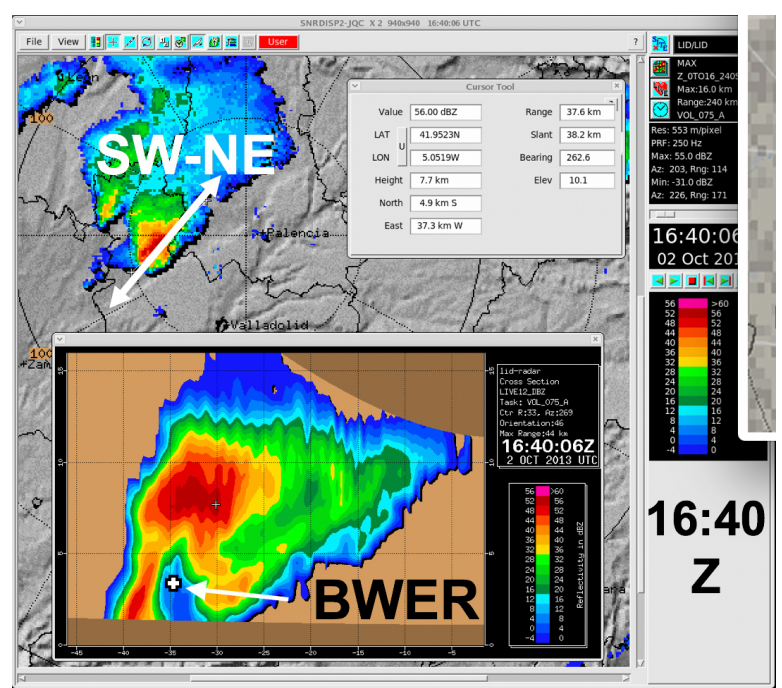

Figura 7. BWER vertical. 


\subsection{BWER-Horizontal}

Aquí, la región de eco débil acotada (BWER) es analizada en la horizontal en vez de en el plano vertical. A diferencia de la BWER-V, en la BWER horizontal la zona de eco débil sí está completamente rodeada por ecos de reflectividades superiores. En la imagen de las 16:30 ecos de 16-20 dBZ están completamente rodeados por ecos superiores a $40 \mathrm{dBZ}$, y de forma similar ocurre en la imagen de las 19:40, donde además se aprecia la característica $V$-Notch en los ecos de color verde. La supercélula cumplía los criterios del modelo de Browning, que postula que el máximo de $\mathrm{Z}$ en niveles medios-altos debe quedar situado encima de la BWER en niveles medios y esta encima del hueco del gancho (o WER) de la supercélula (véanse las figuras $8 \mathrm{a}$ y $8 b$ ).

a)

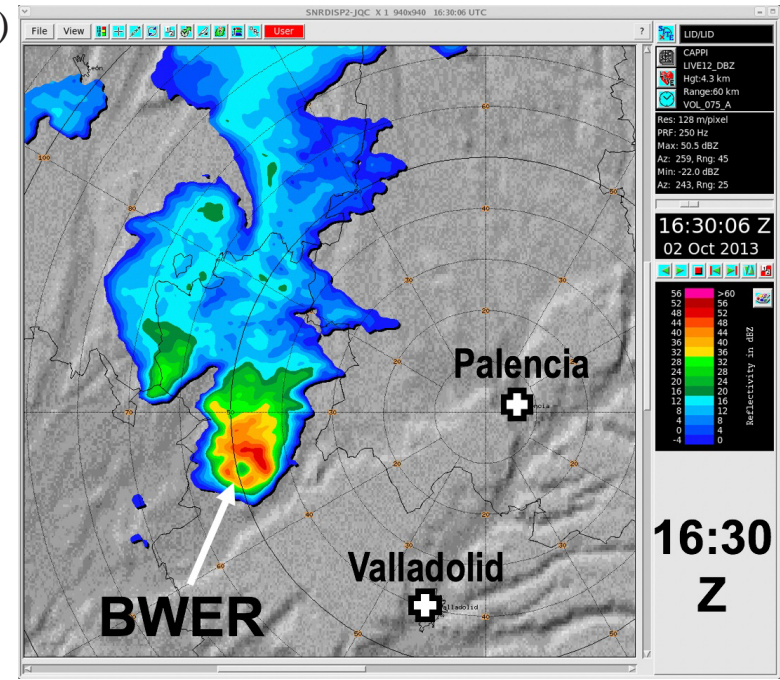

b)

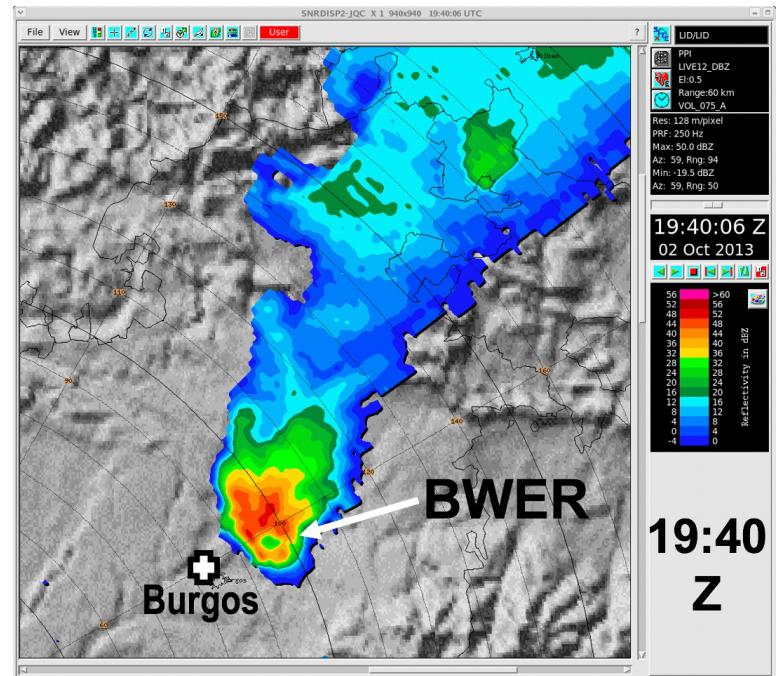

Figura 8. BWER horizontal.

\subsection{Patrón $V$-Notch}

Es un patrón característico en forma de «V» observado en reflectividad, situado en el borde de ataque de la supercélula, corriente abajo del viento en niveles altos. En la supercélula se aprecia en la zona N-NE de la corriente descendente principal (FFD). Este rasgo es una indicación de flujo divergente alrededor de la potente corriente ascendente en rotación. De alguna manera, el mesociclón, se comporta como un obstáculo sólido al flujo incidente sobre él. En nuestro caso, el flujo de niveles medios y altos incidía en la cara S-SW (barlovento), divergiendo y dividiéndose en dos ramas al N y NE del mesociclón (sotavento) (véanse las figuras $9 \mathrm{a}, 9 \mathrm{~b}$ y $9 \mathrm{c})$.

a)

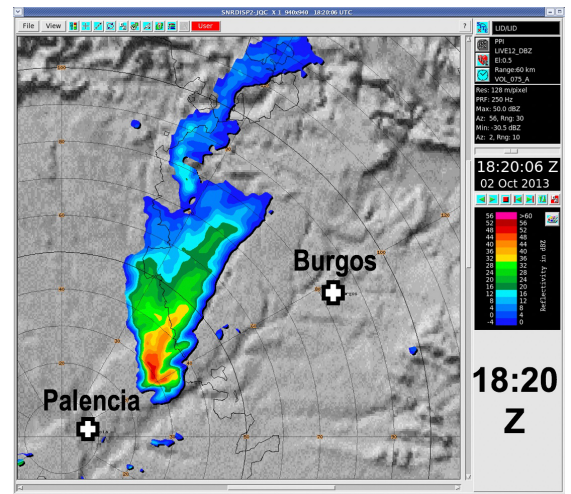

b)

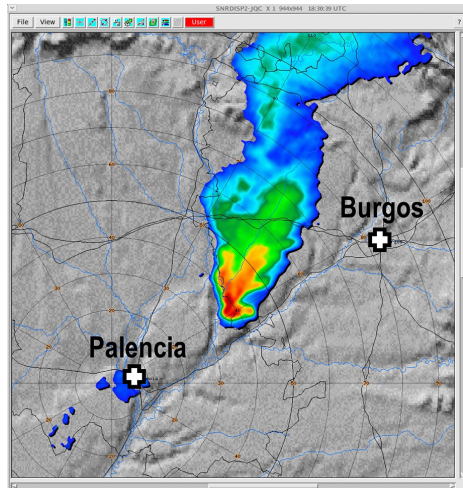

c)

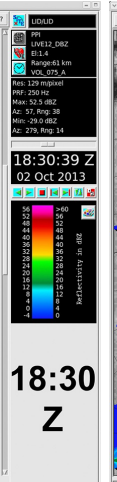

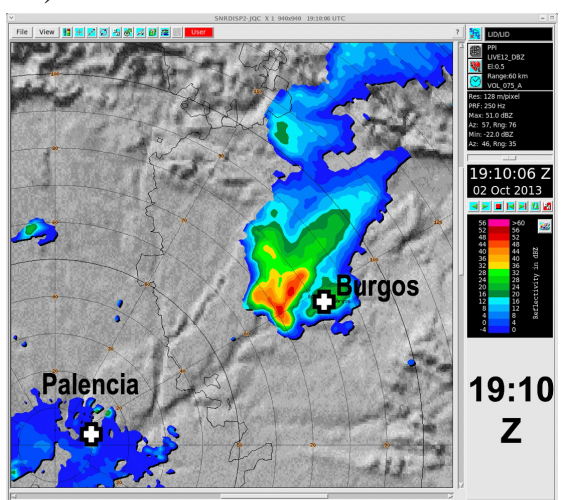

Figura 9 


\subsection{Mesociclón - Viento Doppler}

Identificar un mesociclón en el producto viento Doppler del radar es la «prueba de oro» que nos confirma si la estructura observada es o no una supercélula. Para ello debemos:

1. Detectar dos máximos relativos de vientos opuestos muy cercanos (colores opuestos).

2. El segmento de unión entre esos máximos debe ser perpendicular — o casi—a la radial al punto central donde está el radar (véase la figura 10).

3. Las direcciones asociadas a los máximos nos indicarán si el giro es ciclónico o anticiclónico.

4. El mesociclón debe quedar situado dentro del hueco que deja el «gancho» de reflectividad (WER) y no en otro sitio. Para ello deberán, como hacemos en la figura 10, analizarse siempre por parejas las imágenes de reflectividad y viento Doppler.

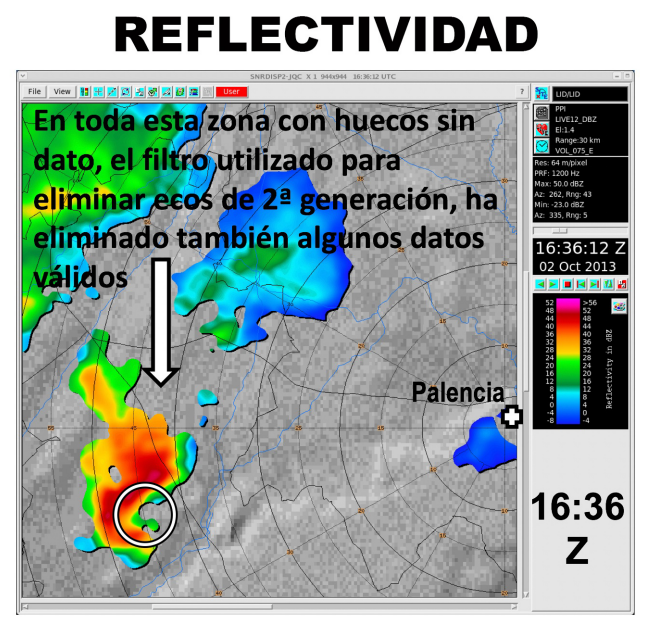

\section{VIENTO DOPPLER}
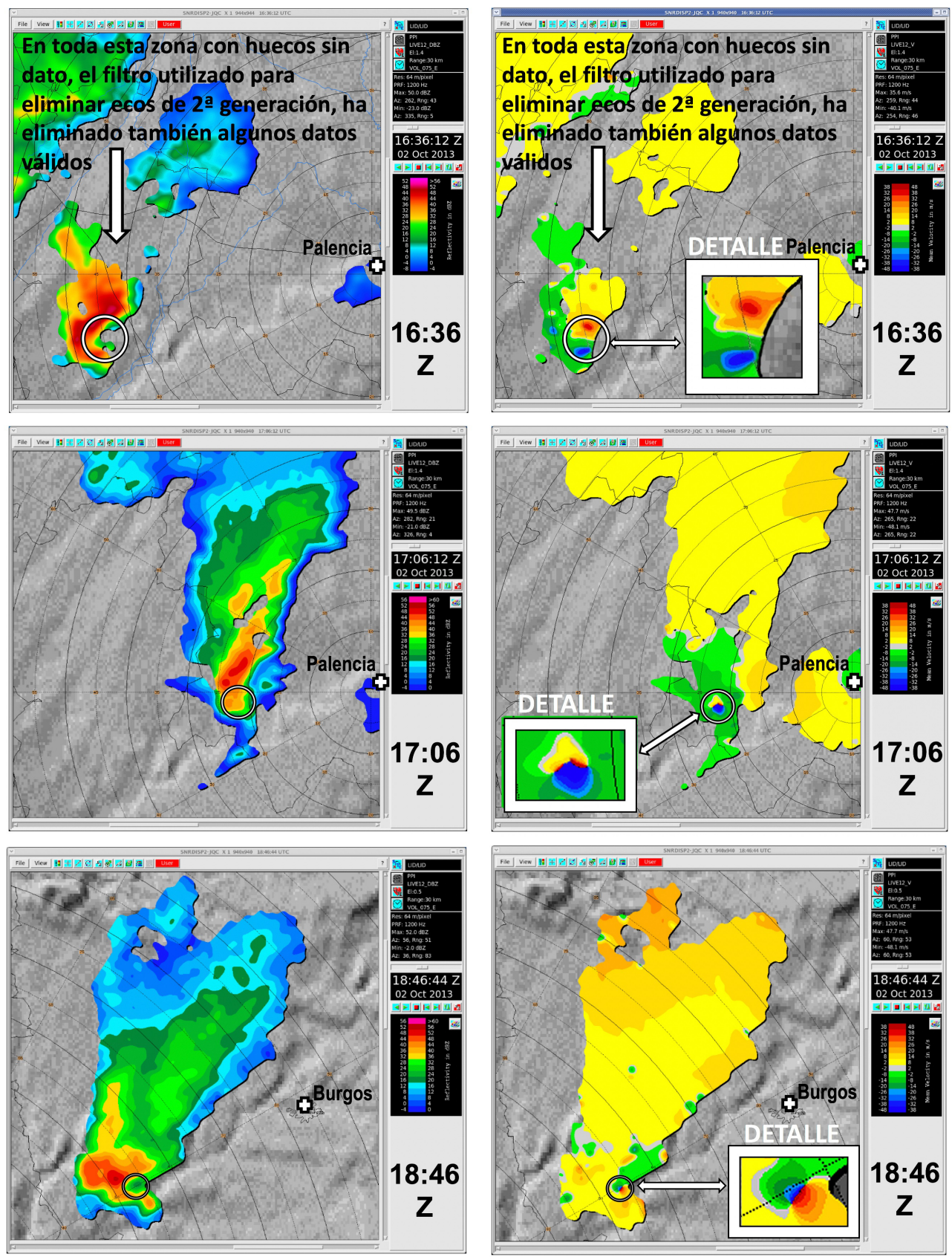

Figura 10 


\subsection{Storm-Splitting}

Muchas de las supercélulas en España provienen de un storm-splitting (SS). En nuestro caso, el SS se produce sobre las 15:30 Z en un entorno con una intensa cizalladura unidireccional, lo que favoreció este tipo de subdivisión celular. A partir de esa hora y lugar, la helicidad relativa a la tormenta (SRH), fue claramente favorable al miembro ciclónico (véanse sondeos). En la figura 11 se ve un corte vertical NW-SE, donde se aprecia el mayor desarrollo del miembro ciclónico o right-mover, a la derecha, respecto al miembro anticiclónico o left-mover, a la izquierda.

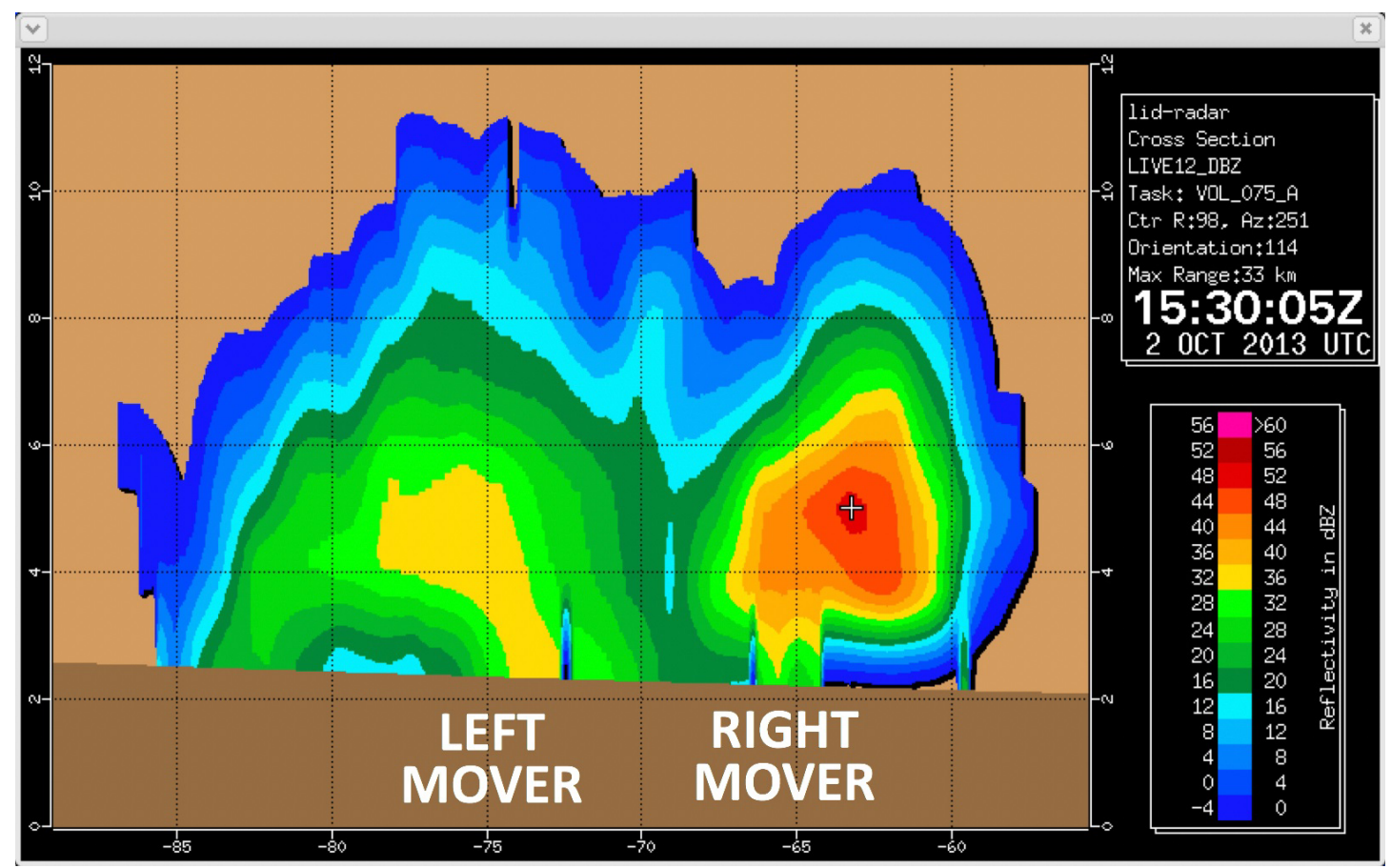

Figura 11

\subsection{Otras características radar}

Además de las características radar mencionadas anteriormente, en las supercélulas también se pueden encontrar otras tales como: a) el flanking-line (figura 12) o línea de torres de cúmulos adyacente a la corriente ascendente en rotación, formada por la convergencia del flujo cálido alimentador del SE y el frente de racha frío de la corriente descendente del flanco trasero o RFD. Para apreciar esta particularidad hemos bajado el umbral mínimo de reflectividad a $8 \mathrm{dBz}$; b) el VIL (figura 13), con valores de hasta $37 \mathrm{~mm}$, muy subestimado por el problema de descalibración del radar de Palencia mencionado anteriormente; y los c) los echotops (figura 14), que fueron bastante altos para la época del año en que ocurrió el episodio, casi $15 \mathrm{~km}$.

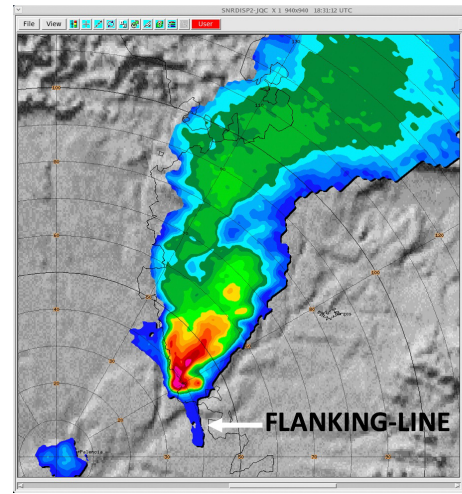

Figura 12

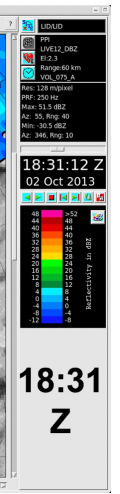

$18: 31$

(1)

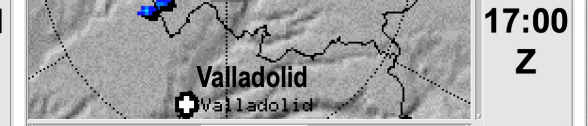

Figura 13

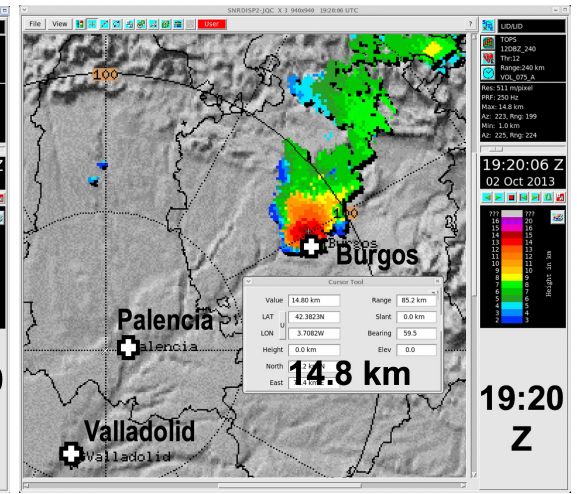

Figura 14 


\section{CONCLUSIONES}

1. El análisis de la estructura radar de la tormenta ha permitido identificar los patrones de las estructuras típicas de las supercélulas: Hook-Echo, FFD, RFD, mesociclón, WER, BWER, V-NOTCH, storm-splitting, flanking-line, etc. Por lo que se puede afirmar, sin lugar a duda, que se trató de una supercélula ciclónica.

2. La duración de la supercélula fue de unas 7 horas, que según las estadísticas disponibles es muy superior a la duración media para estas estructuras, por lo que la tormenta queda caracterizada como supercélula de larga duración. La información de retorno recibida de la formación de un tornado, completa la caracterización como supercélula tornádica de larga duración.

3. La extrema severidad de los efectos en superficie a los que dan lugar estas tormentas nos hace concluir que su identificación en la vigilancia es fundamental, por lo que es importante avanzar en el desarrollo de herramientas de vigilancia y nowcasting basadas en el radar que ayuden en su rápida detección. Sería conveniente estudiar cuál debería ser la mejor estrategia de barrido del radar que permita optimizar la detección de mesociclones, que en cualquier caso debería pasar por disponer de más elevaciones de viento Doppler que en la actualidad.

\section{REFERENCIAS}

Bunkers, M. J., Huelmfelt, M. y Smith, P., 2005. An Observational Examination of Long-Lived Supercells. Part I: Characteristics, Evolution, and Demise. Part II: Enviromental Conditions and Forecasting. Wea. F., 21.

COMET/MetEd 2010-2017. Radar Signatures for Severe Convective Weather.

GómEz, M. A., 2008. Estudio basado en datos de sondeos atmosféricos, del ambiente pre-convectivo asociado a 25 supercélulas en la Península Ibérica, periodo 2001-2007. Magister INM-UCM.

LEMON, L. R., 1980. Severe Thunderstorm Radar Identification Techniques and Warning Criteria. NOAA Tec. Memo. NWS NSSFC-2, Kansas City, 67 pp.

Markowski, P. y Richardson, Y., 2010. Mesoscale Meteorology in Midlatitudes. Wiley-Blackwell. ISBN: 978-0470742136. 430 pp.

Quirantes, J. A., RIESCO, J. y NúÑEZ, J. A., 2014. Características básicas de la supercélulas en España. AEMET. Publicación en línea. http://www.aemet.es/es/conocermas/recursos_en_linea/publicaciones_y_estudios/ publicaciones/.

QuiRANTES, J. A., 2012. Introducción a las supercélulas. Taller de convección para predictores del Centro Nacional de Predicción. Presentación interna. AEMET.

Quirantes, J. A., 2007. Supercélulas. Identificación visual y características radar. XIII Jornades de Meteorologia «Eduard Fontseré». ACAM. 